\title{
Modelización de tableros de obras de paso de luces cortas ejecutados con placa alveolar pretensada
}

\author{
Short span prestressed hollow core slabs bridge decks modeling \\ $\underline{\text { V. Moreno-Padilla }}^{(*)}$, L. Albajar-Molera ${ }^{(*)}$
}

\section{RESUMEN}

Los métodos tradicionales de emparrillado plano utilizados para el cálculo de tableros conformados con placa alveolar pretensada no aportan suficiente precisión para determinar la influencia de fenómenos tales como la torsión sobre las placas o el rasante entre la capa de reparto y la propia placa. Se propone un nuevo método de modelización híbrido que permite cuantificar de forma más realista el mecanismo resistente de este tipo de tableros. La aplicación del método sobre un tablero tipo ha permitido obtener unos resultados que demuestran la buena correlación de las predicciones numéricas con el comportamiento observado en la realidad.

Palabras clave: Puentes; placa alveolar; juntas longitudinales; torsión.

\section{ABSTRACT}

Traditional methods of plane grilled modeling used for the calculation of prestressed hollow core slab bridge decks not provide sufficient accuracy to determine the influence of phenomena such as slabs torsion or shear between the interface slab-toping. A new hybrid modeling approach is proposed. It allows more realistically quantify the resistant mechanism of this type of decks. The application of the method on a typical deck has yielded results that show the good correlation between the numerical predictions and the behavior observed in reality.

Keywords: Bridges; hollow core slab; longitudinal joints; torsion.

${ }^{(*)}$ ETS Ingenieros de Caminos, Canales y Puertos - Universidad Politécnica de Madrid, Spain. Persona de contacto/Corresponding author: vmorenopadilla@yahoo.es (V. Moreno-Padilla)

Cómo citar este artículo/Citation: Moreno-Padilla, V., Albajar-Molera, L. (2014). Modelización de tableros de obras de paso de luces cortas ejecutados con placa alveolar pretensada. Informes de la Construcción, 66(EXTRA-1): moo9, doi: http://dx.doi.org/10.3989/ ic.13.095.

Licencia / License: Salvo indicación contraria, todos los contenidos de la edición electrónica de Informes de la Construcción se distribuyen bajo una licencia de uso y distribución Creative Commons Reconocimiento no Comercial 3.o. España (cc-by-nc). 


\section{INTRODUCCIÓN}

La industrialización de componentes y sistemas constructivos en el ámbito de la edificación residencial hacen que la placa alveolar sea un producto altamente interesante para su empleo en forjados (1). No obstante, el hecho de que la prefabricación haya dejado de ser cerrada en cuanto a la rigidez de las formas y procesos de fabricación (2), permite adaptar los elementos prefabricados para cada caso particular ya sea en obra civil, industrial o edificación residencial. Surge por tanto de forma natural la posibilidad de extrapolar el uso de la placa alveolar a ámbitos no tradicionales.

La aplicación de placas alveolares pretensadas en tableros de obras de paso de carreteras de luces cortas es una práctica que presenta una creciente demanda debido a la posibilidad de aprovechar las ventajas del sistema.

Tradicionalmente se ha supuesto que el comportamiento de este tipo de tableros es inadecuado para hacer frente a sobrecargas móviles de tráfico por dos motivos fundamentalmente. Las placas alveolares no tienen apenas capacidad resistente a torsión y, la junta existente entre capa de reparto y placas alveolares es incapaz de transmitir las tensiones rasantes porque, en general, no se puede colocar armadura de cosido entre ambos elementos.

A pesar de las posibles causas de fallo citadas anteriormente, el comportamiento en servicio observado en este tipo de tableros es más que satisfactorio.

La explicación de la aparente contradicción entre las predicciones teóricas y las observaciones reales puede obedecer a dos razones fundamentales. Por una parte, técnicas de modelización incapaces de representar los elementos y variables de gobierno de este tipo de tableros pueden conducir a la obtención de esfuerzos distorsionados que no se corresponden con la realidad del mecanismo resistente. Por otra, la intensidad de tráfico pesado que en general soporta este tipo de tableros no es muy elevada porque la ubicación de los mismos suelen ser entornos alejados de vías principales de comunicación tales como zonas rurales o carreteras de montaña. Esto hace pensar que la magnitud de los esfuerzos solicitantes en servicio no alcanza el umbral de diseño prescrito en la normativa puesto que los típicos ejes trident son poco frecuentes en redes secundarias. Aún así, no parece razonable establecer la hipótesis de que estas estructuras funcionan por la aleatoria y aparentemente favorable casuística de restricción de tráfico a que están sometidas.

La modelización tradicional de este tipo de estructuras se ha basado en el método del emparrillado plano (3). Tanto las placas alveolares como la capa de reparto se definen mediante elementos de tipo barra y las juntas entre placas se representan mediante rótulas, o mejor dicho, cuasi-rótulas que transmiten los cortantes verticales y momentos flectores muy bajos (4).

Como alternativa al método del emparrillado se propone un método de modelización híbrido que contiene elementos finitos de tipo lámina para representar la capa de reparto y elementos finitos de tipo barra para representar las placas alveolares. Ambos elementos se conectan de forma rígida mediante la imposición de condiciones de compatibilidad. Las juntas longitudinales entre placas se representan como elementos de contacto no lineales.
Se analiza un tablero tipo mediante este método y se pone de manifiesto que los esfuerzos de torsión sobre las placas quedan prácticamente reducidos a cero gracias al excelente trabajo de la placa de reparto y a la consideración en el modelo del efecto del espesor del las placas alveolares (5). Se corrobora por tanto el buen comportamiento observado en la práctica. El método proporciona una herramienta para futuros diseños adaptados a mayores acciones concentradas.

\section{MORFOLOGÍA BÁSICA DE LOS TABLEROS DE PLACA ALVEOLAR}

Los tableros están formados por dos elementos fundamentales. Placa alveolar de hormigón pretensado y capa de reparto de hormigón armado.

Los elementos de la superestructura no difieren en general del resto de tipos de tableros. Pretiles, capa de rodadura, impostas, iluminación y drenaje pueden asimilarse a los habitualmente empleados en otros tipos de obras de paso.

Las secciones tipo (Figura 1) suelen tener del orden de 8 a 10 $\mathrm{m}$ de anchura para poder albergar cómodamente dos carriles con anchuras variables entre 2 y $3 \mathrm{~m}$ habitualmente y una o dos aceras de aproximadamente $1 \mathrm{~m}$ de anchura además del espacio para los elementos de contención.

Los estribos del puente pueden ser ejecutados in situ o prefabricados. En este último caso se pueden emplear igualmente placas alveolares pretensadas en posición vertical para contención de tierras. También es común el empleo de escamas para formar macizos de suelo reforzado.

Las disposiciones constructivas habituales suelen ser tramos isostáticos únicos con luces aproximadas de hasta $15 \mathrm{~m}$. Los cantos habituales de las placas suele estar entre 35 y $50 \mathrm{~cm}$ y los espesores de la capa de reparto varían entre 10 y $20 \mathrm{~cm}$ (6). No se puede hablar en principio de relaciones canto/luz como tales puesto que en muchas ocasiones se emplea el mismo canto de placa para un rango de luces determinado. Estas dimensiones suelen ser suficientes para salvar pequeños cauces, caminos o incluso carreteras secundarias con cunetas $\mathrm{y}$ arcenes.

En el caso de disponer varios vanos las pilas suelen adoptar la configuración de pórticos o tabiques para recibir a las placas mediante apoyos continuos evitando de esta forma flexiones transversales importantes en un elemento que carece de armadura transversal. Los apoyos se materializan mediante bandas elastoméricas corridas sin zunchar. También se pueden disponer una serie de apoyos discretos bajo cada placa.

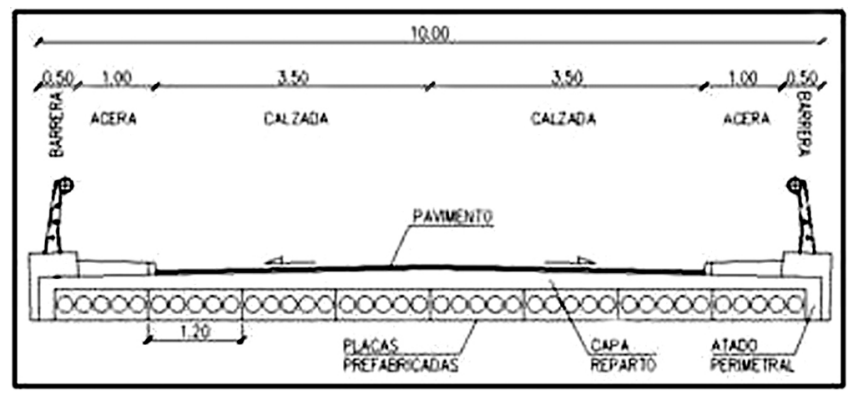

Figura 1. Sección tipo. 
La disposición de varios vanos no implica generalmente continuidad estructural sino únicamente continuidad en la rodadura. La capa de reparto es parcialmente desconectada de las losas.

La técnica empleada en el caso de la ejecución de tableros de obras de paso de carreteras es herencia directa del ámbito de los forjados de edificios de habitación. Sobre los estribos previamente construidos se disponen una serie de placas yuxtapuestas longitudinalmente según el eje de la obra. Sobre las placas se coloca una capa de reparto, de espesor adecuado, de hormigón vertido in situ que, a priori, tiene una doble misión. Por una parte se encarga de transmitir transversalmente entre placas los esfuerzos provocados por acciones verticales consiguiendo de esta forma un diafragma de reparto horizontal como si de un tablero de vigas se tratase; por otra parte actúa como diafragma horizontal frente a acciones horizontales garantizando de esta forma el trabajo uniforme del conjunto. El espesor adecuado de la capa de reparto permite la eliminación de elementos accesorios de arriostramiento.

\section{MODELIZACIÓN TRADICIONAL DE TABLEROS DE PLACA ALVEOLAR PRETENSADA}

A pesar de los medios disponibles en la actualidad y de la posibilidad de representar efectos tales como el espesor de las placas dentro de un forjado o tablero, el método del emparrillado (3) sigue estando en plena vigencia. Cada una de las placas alveolares se representa mediante dos vigas longitudinales (semisección) más la parte tributaria de la capa de reparto (1). A estas barras se les asignan las características de áreas e inercias a flexión y torsión correspondientes. Las barras transversales representan a la capa de reparto y suele haber dos tipos. Barras transversales interiores situadas entre las dos barras longitudinales de cada placa que representan transversalmente a la placa más la capa de reparto y barras transversales exteriores que representan la junta entre placas.

A las barras transversales interiores se les asignan las áreas e inercias transversales del conjunto mientras que a las barras transversales exteriores sólo se les asignan las propiedades de la capa de reparto. De esta forma se recoge en cierta manera el efecto del debilitamiento que supone la junta entre placas y, aunque no llega a ser una rótula tal y como prescriben las normativas, es en cierto modo un punto débil para la flexión y el cortante.

De este tipo de modelos se obtienen de forma directa los esfuerzos principales de flexión y cortante tanto longitudinales como transversales, esfuerzos rasantes y cortantes en juntas. Los esfuerzos de torsión hay que procesarlos y trasladarlos al centro de gravedad de la sección compuesta.

Respecto a estos últimos hay que decir que el hecho de no poder representar el efecto del espesor de la placa (5) se traduce en la sobreestimación de los mismos. Esta sobreestimación puede provocar que la solicitación a torsión de la placa sea superior, al menos teóricamente, a la baja capacidad resistente de la misma. Este hecho ha sido el que tradicionalmente ha provocado el rechazo respecto al empleo de placas alveolares pretensadas en tableros de obras de paso. Se plantea por tanto la necesidad de incorporar los últimos avances en el campo de la modelización para poder establecer magnitudes de las solicitaciones más fiables.
Junto a la débil capacidad resistente a torsión existe otro efecto que hace presuponer un comportamiento inadecuado de este tipo de tableros; la imposibilidad de coser físicamente la junta entre el elemento prefabricado y la capa de reparto. En teoría, los cálculos predicen un fallo por rasante en los extremos del tablero motivado por las acciones exteriores además de los efectos reológicos.

\section{MODELIZACIÓN DE FORJADOS DE PLACA ALVEOLAR PRETENSADA CONSIDERANDO EL ESPESOR}

Antes de desarrollar un modelo integral que represente todos los elementos del tablero conviene avanzar en la modelización precisa de la placa alveolar, junto al efecto de su espesor, y de las juntas longitudinales. Posteriormente se podrá incluir la capa de reparto.

Una placa alveolar puede ser representada perfectamente mediante una serie de barras longitudinales dispuestas según la directriz de la misma. Un forjado puede ser representado por una serie de barras yuxtapuestas separadas la distancia entre ejes de placas.

Las placas alveolares están en la práctica conectadas entre sí mediante juntas longitudinales que se hormigonan in situ. Normalmente, las juntas están fisuradas y tradicionalmente se asume que actúan como rótulas (7). Un avance importante sobre la modelización habitual de las juntas mediante rótulas se recoge en los trabajos de Lundgren que se describen a continuación.

La distribución de las cargas aplicadas sobre las placas introduce momentos torsores sobre las mismas que a su vez introducen fuerzas de contacto horizontales sobre las juntas longitudinales (Figura 2) (8).

Basándose en dichas observaciones, Lundgren (5) establece la siguiente hipótesis. Las fuerzas de contacto horizontales que aparecen en las juntas longitudinales provocan un momento torsor de sentido contrario al que provocan las acciones exteriores precisamente porque las juntas entre placas están hormigonadas. De esta manera, la magnitud el esfuerzo torsor que provocan las acciones exteriores se ve minorado por el efecto favorable del momento torsor que provocan las fuerzas de contacto entre las placas. Por tanto, el efecto del espesor tiene una gran influencia sobre el comportamiento de los forjados y es de vital importancia su inclusión en los modelos de cálculo.

El sistema de modelización de placas alveolares que propone Lundgren define la sección transversal de cada placa

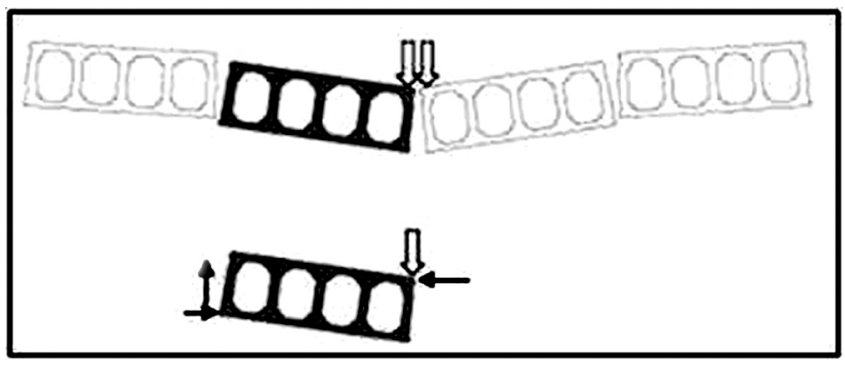

Figura 2. Mecanismo de distribución transversal. Cortesía de Lundgren. 


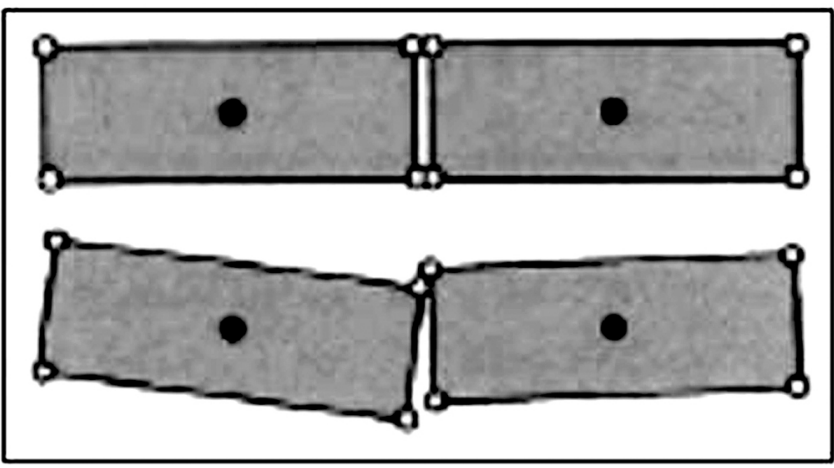

Figura 3. Modelización de placas alveolares. Cortesía de Lundgren.

mediante un único elemento de tipo barra alineada con la directriz. Además, una serie de nodos llamados nodos esclavos se posicionan en las esquinas de la sección transversal (Figura 3). Estos nodos se unen a los nodos de la directriz de forma que, de cara a las deformaciones y giros de la sección transversal, se asumen dos hipótesis básicas de funcionamiento; Navier-Bernouilli (planeidad de secciones) y Saint Venant (torsión sin distorsión).

\section{PROPUESTA DE MODELIZACIÓN HÍBRIDA}

Vistas las posibles carencias del método del emparrillado plano para representar el trabajo real de torsión de las placas, se plantea un nuevo método de modelización para este tipo de tableros basado en las siguientes premisas.

1) Las placas alveolares se representan mediante elementos finitos de tipo barra.

2) Las juntas longitudinales entre placas se representan mediante elementos de contacto no lineales.

3) La capa de reparto se representa mediante elementos finitos de tipo lámina.

Este tipo de modelización es novedoso en el sentido de que incorpora los avances de Lundgren estudiados en casos sin capa de reparto a un caso donde sí existe dicha capa. Las especiales características del modelo exigen de una conexión rígida entre los elementos descritos de forma que se cumplan las hipótesis de Navier-Bernouilli y Saint-Venant. Como aplicación práctica se ha modelizado un tablero (Figura 4).

El tablero está compuesto por ocho placas alveolares de 120 $\mathrm{cm}$ de ancho y $40 \mathrm{~cm}$ de canto. La luz es de $12 \mathrm{~m}$. Las características mecánicas de las placas se muestran en la Tabla 1. El eje $\mathrm{X}$ es horizontal y el eje $\mathrm{Z}$ es vertical ambos pasando por el centro de gravedad de la sección.

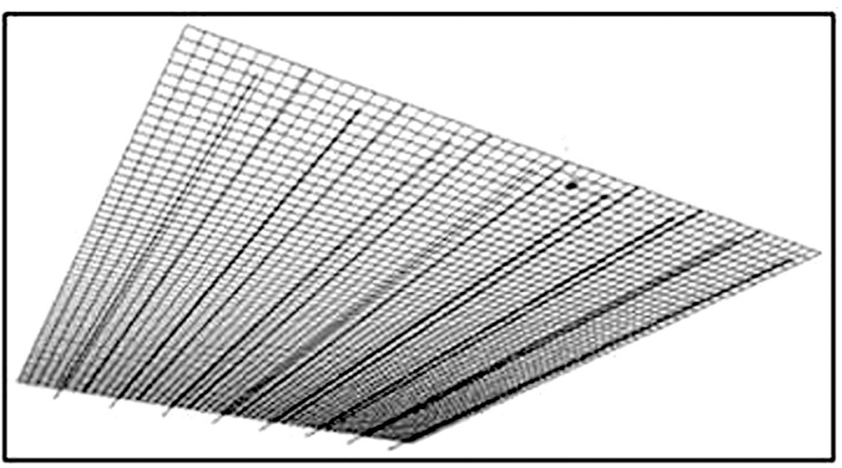

Figura 4. Vista general inferior del modelo.
Tabla 1. Características de las placas.

\begin{tabular}{|l|c|}
\hline Canto de placa $(\mathrm{mm})$ & 400 \\
\hline fck $(\mathrm{Mpa})$ & 40 \\
\hline fct,k (Mpa) & 2,45 \\
\hline $\mathrm{E}(\mathrm{Mpa})$ & 30891 \\
\hline $\mathrm{A}\left(\mathrm{m}^{2}\right)$ & 0,2108 \\
\hline $\mathrm{Ix}\left(\mathrm{m}^{4}\right)$ & $4,4310-3$ \\
\hline $\mathrm{Iz}\left(\mathrm{m}^{4}\right)$ & $2,8010-2$ \\
\hline $\mathrm{It}\left(\mathrm{m}^{4}\right)$ & $9,0310-3$ \\
\hline $\mathrm{Ap}\left(\mathrm{mm}^{2}\right)$ & 976,5 \\
\hline
\end{tabular}

Las placas se disponen simplemente apoyadas en los extremos de forma que un extremo tiene impedidos los tres desplazamientos y dos de los giros permitiendo el giro principal de flexión longitudinal. El extremo opuesto tiene las mismas coacciones pero además permite el desplazamiento longitudinal materializando de esta forma un apoyo móvil. La directriz de cada placa se discretiza mediante 60 barras de $20 \mathrm{~cm}$ de longitud. Asociados a cada nodo de la directriz se colocan cuatro nodos esclavos de forma que el conjunto de los cinco nodos cumple simultáneamente las dos condiciones de compatibilidad mencionadas.

Los elementos elegidos para la discretización de la directriz son barras de dos nodos que utilizan una formulación tridimensional de tipo viga-columna que incluye los efectos de flexión biaxial, torsión, deformación axial y deformaciones por cortante.

Las distancias entre nodos esclavos extremos de placas son de $3 \mathrm{~cm}$ para materializar el ancho de las juntas. Cada dos nodos están conectados por un elemento de conexión que permite desarrollar fuerzas de compresión perpendiculares al plano de la junta. No se permiten tracciones. En las direcciones vertical y longitudinal de la junta se permiten fuerzas tanto de compresión como de tracción.

Las rigideces de los elementos de conexión se muestran en la Tabla 2.

Tabla 2. Características de las juntas.

\begin{tabular}{|l|c|}
\hline Compresión junta $\left(\mathrm{N} / \mathrm{m}^{3}\right)$ & $3 \times 10^{10}$ \\
\hline Tracción junta $\left(\mathrm{N} / \mathrm{m}^{3}\right)$ & 0 \\
\hline Cortante vertical junta $\left(\mathrm{N} / \mathrm{m}^{3}\right)$ & $1 \times 10^{9}$ \\
\hline Cortante longitudinal junta $\left(\mathrm{N} / \mathrm{m}^{3}\right)$ & $1 \times 10^{9}$ \\
\hline
\end{tabular}

La junta representa una no linealidad geométrica. Además, es necesario representar la apertura de la misma de forma que se puedan tener en cuenta los fenómenos de retracción del hormigón vertido in situ, la edad de las placas y el tamaño de la junta. El valor de apertura de junta elegido es de 0,5 mm puesto que representa el límite superior recomendado para que los mecanismos de fricción y acuñamiento se puedan desarrollar (9).

Las ecuaciones constitutivas de los elementos de contacto que representan las condiciones impuestas son:

1) $\mathrm{F}=\mathrm{k}\left(\mathrm{d}+\mathrm{d}_{\text {apertura }}\right)$ si $\mathrm{d}+\mathrm{d}_{\text {apertura }}<\mathrm{O}$

2) $\mathrm{F}=\mathrm{O}$ Resto de casos

La capa de reparto se modeliza mediante elementos finitos de tipo lámina de cuatro nodos con formulación general de Kirchoff. Las características se muestran en la Tabla 3. 
Tabla 3. Características de la capa de reparto.

\begin{tabular}{|l|c|}
\hline Espesor del elemento $(\mathrm{mm})$ & 200 \\
\hline Dimensiones $\left(\mathrm{mm}^{2}\right)$ & $200 \times 200$ \\
\hline fck $(\mathrm{Mpa})$ & 25 \\
\hline E $(\mathrm{Mpa})$ & 27264 \\
\hline
\end{tabular}

\section{BASES DE CÁLCULO}

\subsection{Acciones}

Las acciones empleadas en el modelo son:

Peso propio de placas $(\mathrm{PP})=5,27 \mathrm{kN} / \mathrm{m}$.

Pretensado (P). Se ha introducido como una fuerza exterior de compresión de valor $976,5 \mathrm{kN}$ y un momento negativo de valor -166 kNm en los extremos de las placas; no se han considerado pérdidas.

Peso propio de la capa de reparto $(\mathrm{PPCRI})=6 \mathrm{kN} / \mathrm{m}$ para las placas interiores y PPCRE $=9 \mathrm{kN} / \mathrm{m}$ para las placas exteriores debido al peso de los zunchos de atado.

Resto de carga permanente correspondiente a aceras (RCPA) $=5 \mathrm{kN} / \mathrm{m}^{2}$.

Resto de carga permanente correspondiente a barreras $(\mathrm{RCPB})=10 \mathrm{kN} / \mathrm{m} /$ barrera.

Resto de carga permanente correspondiente a un espesor de pavimento de $5 \mathrm{~cm}(\mathrm{RCPP})=1,2 \mathrm{kN} / \mathrm{m}^{2}$.

Sobrecarga de uso uniforme $(\mathrm{SCU})=4 \mathrm{kN} / \mathrm{m}^{2}$ según IAP-98 (10).

Tren de cargas $(\mathrm{CA})=6 \times 100 \mathrm{kN}$ según IAP-98 (10).

Los valores de cálculo de las acciones para ELU y ELS se han obtenido de IAP-98 (10).

\subsection{Comportamiento de los materiales}

Los materiales se han caracterizado mediante su módulo de deformación longitudinal y transversal. Las secciones empleadas en los cálculos son brutas y el comportamiento es elástico y lineal en todo momento. Este planteamiento es compatible con la filosofía de los estados límite para el cálculo de esfuerzos y la posterior comprobación en servicio o rotura.

No se han considerado efectos reológicos ni térmicos. No obstante, en la actualidad se están estudiando dichos efectos en modelos de mayor complejidad.

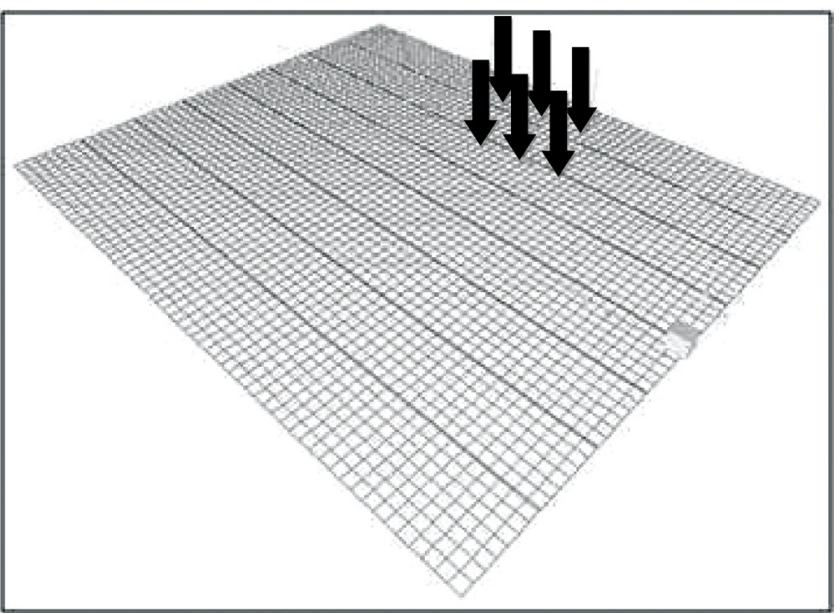

\subsection{Proceso constructivo}

La secuencia de fases ha sido:

Fase-1. Pretensado (P) + (PP). Sección simple.

Fase-2. Montaje de Superestructura (RCP). Sección compuesta.

Fase-3. Sobrecarga de uso (SCU). Sección compuesta.

Fase-4A. Carro en centro de vano excéntrico (CA_CV). Sección compuesta.

Fase-4B. Carro en apoyo excéntrico (CA_AP). Sección compuesta.

Por simplicidad sólo se muestran los gráficos (Figura 5) correspondientes a la hipótesis del carro CA. La numeración de las placas es 1 a 8 siendo la placa 1 la más alejada del carro (CA).

\section{RESULTADOS ELS}

\subsection{Fisuración por tensiones normales}

El siguiente gráfico (Figura 6) muestra el incremento tensional de la armadura de pretensado por efecto de las acciones exteriores para la combinación frecuente. Los valores positivos indican tracciones. Puede comprobarse que el pretensado de las placas extremas está por debajo de 200 Mpa por lo que la abertura de fisura no supera los 0,2 mm (4). Las placas interiores se encuentran en descompresión.

\section{RESULTADOS ELU}

Se analizan los esfuerzos que tradicionalmente han supuesto un impedimento para su aplicación. Cortante, torsión, cortante longitudinal en juntas y rasante entre la capa de reparto y placas.

\subsection{Cortante}

La comprobación del ELU de cortante se hace conforme al artículo 44 de EHE-08 (4). El gráfico adjunto (Figura 7) muestra la distribución de cortantes en las placas del modelo para la combinación de hipótesis correspondiente a la actuación de peso propio (PP), pretensado (P), resto de carga permanente (RCP), sobrecarga de uso extendida a todo el tablero (SCU) y carro excéntrico a un canto útil del apoyo (CA_AP).

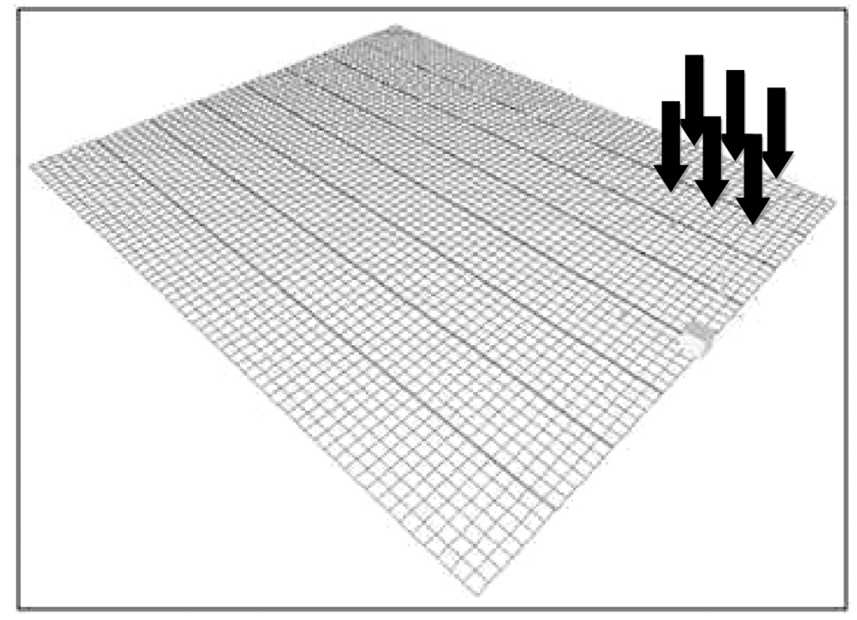




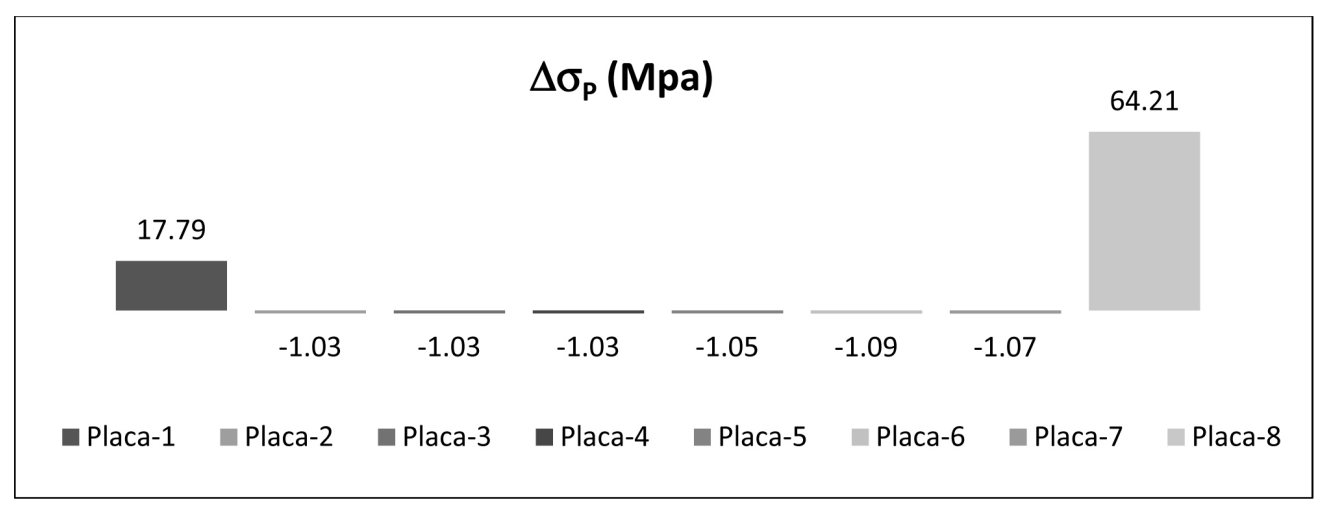

Figura 6. Incrementos tensionales en la armadura de pretensado.

También se ha representado la capacidad del alma $\mathrm{Vu}_{2}$ y la reducida por efecto del torsor $\mathrm{Vu}_{2 \mathrm{n}}$.

En rigor, la capacidad $\mathrm{Vu}_{2}$ mostrada no sería una línea recta sino curva en función de la zona (fisurada o no) donde se encuentre la sección en estudio; pero en este caso no se pretende evaluar la capacidad de cada sección a cortante sino comparar con un valor límite.

Las placas 6, 7 y 8 acusan fuertemente la presencia del carro mostrando tres saltos en la ley de variación del cortante coincidentes con la posición de las cargas. Puede verse cómo a partir de la placa 5 se empieza a notar el efecto del carro como consecuencia de haber introducido en el modelo los elementos de conexión entre placas (juntas longitudinales). La distribución de cortantes sobre los apoyos no es muy uniforme, a pesar de la influencia de la capa de reparto, debido a la presencia del carro en las proximidades.

\subsection{Torsión}

El mecanismo resistente a torsión contenido en EHE-o8 (art. 45) responde a un esquema de bielas y tirantes basado en la obtención de una sección hueca equivalente. Precisa de tres elementos para su formación. Dos familias de armadura pasiva, cercos transversales y barras longitudinales, colocadas en el perímetro de la sección hueca equivalente para la materialización de los tirantes, y bielas de hormigón alojadas en las paredes de dicha sección.

Los elementos de una placa alveolar necesarios para la formación de la sección hueca equivalente son las tablas superior e inferior y las almas exteriores. Las bielas se pueden materializar fácilmente a través de las tablas superior e inferior así

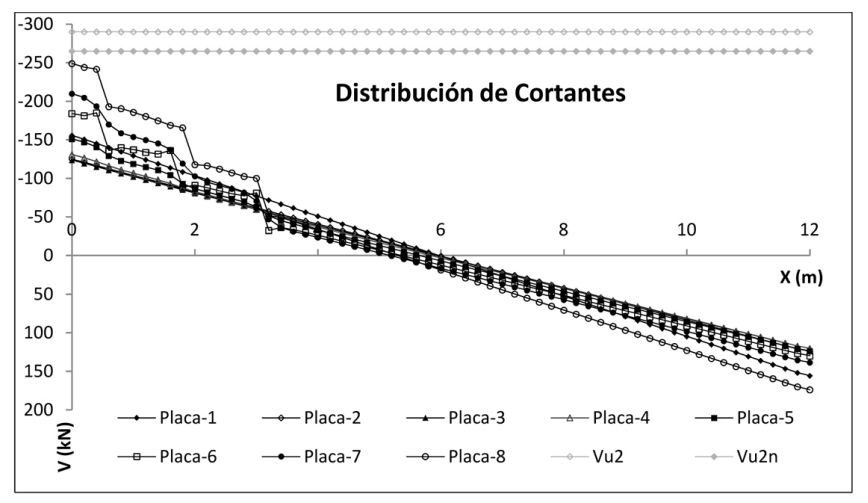

Figura 7. Distribución de cortantes en placas. como de las almas exteriores. Los alambres o tendones de pretensado pueden hacer las funciones de tirantes longitudinales aunque incumplen una condición básica para la formación del mecanismo; no están distribuidos uniformemente sobre el perímetro de la sección. Los tirantes transversales no se pueden materializar por la imposibilidad de colocar cercos cerrados sobre el perímetro de la pieza durante el proceso de fabricación. Únicamente se puede confiar en la resistencia a tracción del hormigón para su formación pero esto limita fuertemente la capacidad resistente del mecanismo a torsión además de ser incongruente con las prescripciones de EHE-08 (4).

El mecanismo resistente a torsión de una placa alveolar no se puede calibrar con los modelos de EHE-08 puesto que cuando aparece la primera fisura sobre la tabla superior de la placa puede decirse prácticamente que la placa está rota. Es cierto que existe una capacidad residual en estado de rotura pero no es fácilmente cuantificable. Por ello parece más lógico estudiar el comportamiento a torsión dentro del dominio elástico, es decir, en los instantes previos a la aparición de la primera fisura. Esto es conocido como estado de prefisuración (11).

Para obtener el momento torsor de fisuración de una placa alveolar pueden utilizarse básicamente dos estrategias.

La primera está basada en la obtención de los flujos de tensiones tangenciales que provocan los momentos torsores sobre las paredes de la sección hueca equivalente (4). Esto se consigue fácilmente mediante resistencia de materiales multiplicando el módulo resistente a torsión de la placa por el valor de la resistencia media a tracción del hormigón. Este método no tiene en cuenta el efecto favorable del pretensado de la placa por lo que el valor obtenido podría resultar muy conservador para secciones situadas en centro de vano donde el pretensado es claramente colaborante. Sin embargo, para secciones próximas a los apoyos donde el pretensado, aparte de no estar plenamente desarrollado, no colabora en la tabla superior de la placa, sí puede resultar conveniente emplear este tipo de formulación. De hecho, numerosos ensayos de placas alveolares a torsión pura (12) muestran muy buena correlación entre los valores predichos por este método y los observados en la práctica.

La segunda estrategia está basada en la obtención del momento torsor de prefisuración mediante formulaciones que tengan en cuenta no sólo la resistencia a tracción del hormigón sino también el efecto del pretensado. Como ejemplo puede ponerse la norma ACI-318 cuya fórmula se expresa a continuación. 


$$
T_{f i s}=2 h_{e} A_{e} f_{c t} \sqrt{1+\frac{\sigma_{x}}{f_{c t}}}
$$

Donde $A_{e}$ y $h_{e}$ son los valores del área y espesor de la sección hueca equivalente, $f_{c t}$ es la resistencia a tracción del hormigón y $\sigma_{x}$ representa el valor de la tensión de pretensado sobre la sección en estudio.

Aplicando ambas formulaciones para las placas utilizadas en el presente trabajo se obtiene un valor del momento torsor de fisuración de $105 \mathrm{kNm}$ para el caso de la formulación de resistencia de materiales y un valor de $117 \mathrm{kNm}$ para la formulación ACI-318 sin tener en cuenta el efecto favorable del pretensado.

Como puede observarse, ambos valores están próximos entre sí con una diferencia del orden del $10 \%$. A su vez, el valor del momento torsor de fisuración observado en los ensayos de laboratorio está en el entorno de los $90 \mathrm{kNm}$ lo que ciertamente corrobora la hipótesis respecto al dominio en el cual estudiar la torsión en placas alveolares.

El siguiente gráfico (Figura 8) muestra la distribución de momentos torsores en las placas del modelo para la combinación de hipótesis correspondiente a la actuación de peso propio (PP), pretensado (P), resto de carga permanente (RCP), sobrecarga de uso extendida a todo el tablero (SCU) y carro excéntrico a un canto útil del apoyo (CA_AP). Con objeto de percibir con mayor precisión el efecto de la torsión sobre cada placa se ha prescindido de representar el valor límite del momento torsor de agotamiento para las placas $(\mathrm{Mt}=8 \mathrm{o} \mathrm{kNm})$.

El gráfico muestra cómo las placas más alejadas de la zona de influencia del carro (placas 1 y 2) apenas reciben el influjo del mismo mientras que las placas 5 y 6 son las más solicitadas. Una vez más se pone de manifiesto el efecto de la inclusión de las juntas longitudinales en el modelo mediante elementos de contacto. Se corrobora la hipótesis de Lundgren (5). El efecto del espesor de las placas hace que los momentos torsores sobre las mismas se vean disminuidos notablemente por la aparición de fuerzas en el contacto entre placas de sentido contrario a las introducidas por las fuerzas exteriores.

Por otra parte, debido al trabajo de la capa de reparto, la magnitud de los esfuerzos torsores es muy reducida y las placas se encuentran por tanto muy lejos de su capacidad de agotamiento. Esta es la principal característica del funcionamiento de los tableros de placa alveolar pretensada. La capa de reparto realiza un excelente trabajo aliviando a las placas de torsiones innecesarias y poniendo de manifiesto, en analogía con los tableros de vigas, que un espesor adecuado de la misma permite afrontar el trabajo de reparto transversal sin necesidad de implicar a las placas. De esta forma se pueden aprovechar éstas para el desempeño fundamental del trabajo de flexión longitudinal.

\subsection{Cortante en juntas longitudinales}

La comprobación del cortante vertical en las juntas se lleva a cabo mediante la aplicación del artículo 44.2.3.6 de EHE-o8 (4). Se tienen en cuenta dos secciones de control. La primera es la compuesta por capa de reparto y tablas superior e inferior de las placas que proporciona valor de $\mathrm{V}_{\mathrm{u}}=92,1 \mathrm{kN} / \mathrm{m}$. La otra sección es la compuesta únicamente por hormigón vertido in situ. En este caso $\mathrm{V}_{\mathrm{u}}=107,1 \mathrm{kN} / \mathrm{m}$. Se toma el valor mínimo para la comprobación.

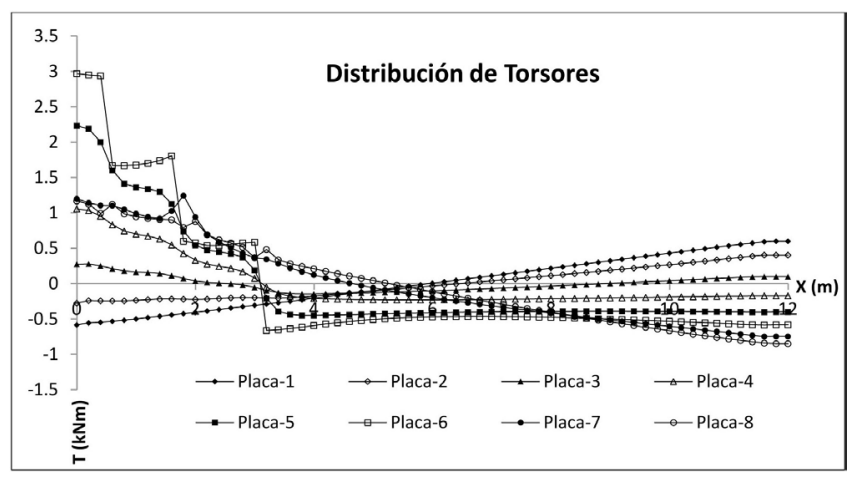

Figura 8. Distribución de torsores en placas.

El gráfico adjunto (Figura 9) muestra la distribución de cortantes verticales en las juntas longitudinales entre placas para la combinación de hipótesis correspondiente a la actuación de peso propio (PP), pretensado (P), resto de carga permanente (RCP), sobrecarga de uso extendida a todo el tablero (SCU) y carro excéntrico en centro de vano (CA_CV).

En la zona de influencia de las cargas puntuales del carro se acusa un incremento de la magnitud del cortante pero no se llega a rebasar la capacidad de la junta en ningún caso.

\subsection{Rasante entre hormigones}

La comprobación de las tensiones rasantes en la junta existente entre placas alveolares y capa de reparto se hace conforme al artículo 47.2.1 de EHE-08 (4) y está basada en la suposición de dos hipótesis. No existe armadura de cosido y la superficie de contacto puede considerarse como de alta rugosidad. Adicionalmente el articulado establece la limitación de reducir en un 50 \% el factor de cohesión entre hormigones cuando las solicitaciones sean de tipo dinámico o de fatiga. Esta cuestión es muy discutible en este tipo de tableros pero aún así se ha tenido en cuenta por ser esta comprobación especialmente sensible para explicar el buen funcionamiento observado en los puentes construidos.

En el gráfico adjunto (Figura 10) se representa la distribución de tensiones rasantes de cálculo en cada punto de la directriz de la placa 7 situada bajo el carro para la combinación de hipótesis correspondiente a la actuación de peso propio (PP), pretensado (P), resto de carga permanente (RCP), sobrecarga de uso extendida a todo el tablero (SCU) y carro excéntrico en centro de vano (CA_CV). También se representa la tensión rasante última soportada por la junta.

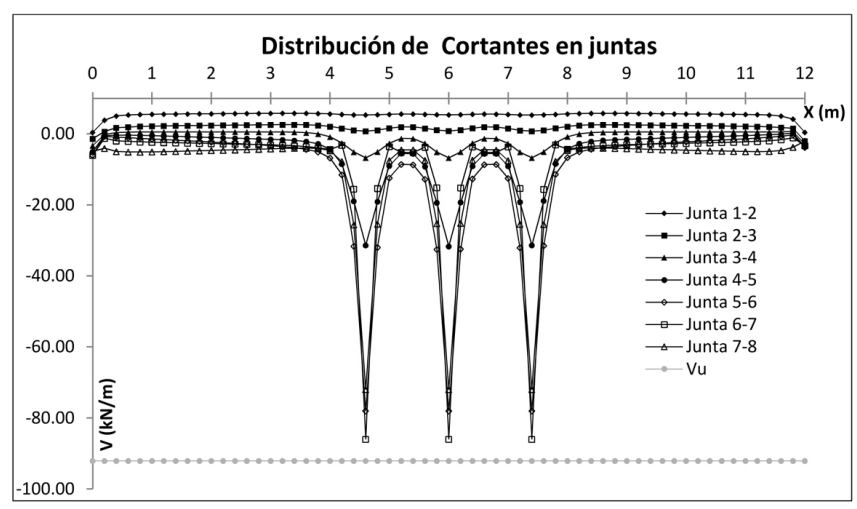

Figura 9. Distribución de cortantes en juntas longitudinales. 


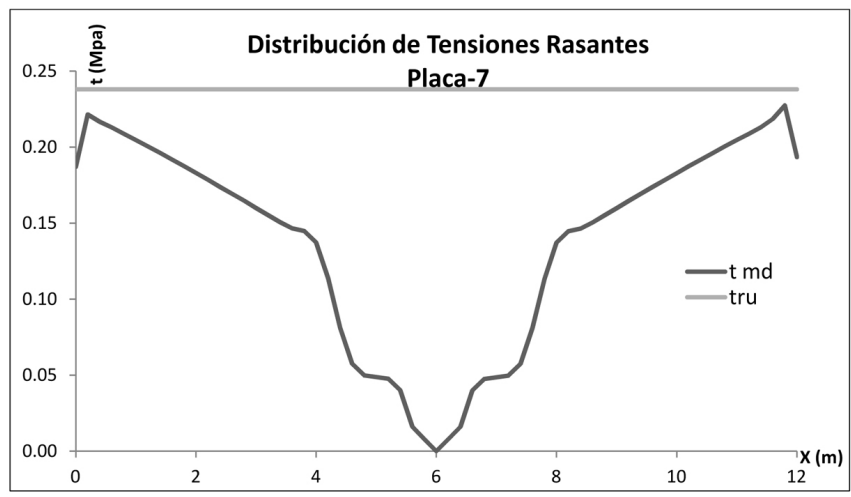

Figura 10. Distribución de tensiones rasantes en placa 7 .

A la vista de los resultados puede decirse que todas las secciones se hallan dentro del dominio de seguridad incluso las zonas próximas a los extremos libres.

El hecho de representar en el modelo los elementos placa alveolar y capa de reparto de manera independiente permite cuantificar de manera directa los esfuerzos rasantes sobre esta última.

\section{CONCLUSIONES Y TRABAJOS POSTERIORES}

1) Los métodos tradicionales de emparrillado plano utilizados para el cálculo de tableros conformados con placa alveolar pretensada no aportan suficiente precisión para determinar la influencia de fenómenos tales como la torsión sobre las placas o el rasante entre la capa de reparto y la propia placa.

2) El modelo de barras (Modelo de Lundgren) permite modelizar un forjado de placas alveolares sin capa de reparto introduciendo el efecto del espesor de las placas con la consecuente repercusión sobre la minimización de los esfuerzos de torsión sobre las placas. También permite representar el comportamiento real de las juntas longitudinales entre placas.
3) Se propone un tipo de modelización denominado híbrido formado por elementos de tipo barra para la representación de las barras y elementos de tipo lámina para la representación de la capa de reparto. Ambos elementos se conectan rígidamente. Las juntas longitudinales se modelizan mediante elementos no lineales de contacto. Dicho modelo se aplica a un tablero de $12 \mathrm{~m}$ de luz formado por 8 placas de $40 \mathrm{~cm}$ de canto sobre las que se coloca una capa de reparto de $20 \mathrm{~cm}$.

4) La predicción obtenida en la comprobación del ELS de fisuración por tensiones normales se corresponde con las observaciones realizadas sobre puentes construidos.

5) Se comprueban determinados ELU que tradicionalmente han puesto en tela de juicio la validez de las placas alveolares para su empleo en tableros (cortante, torsión, cortante en juntas longitudinales y rasante entre hormigones). Todas las comprobaciones resultan satisfactorias.

6) En el caso particular de la comprobación de torsión, se pone claramente de manifiesto que los esfuerzos sobre las placas prácticamente desaparecen gracias a dos factores. La inclusión en el modelo de cálculo de los efectos del espesor de las placas y de la capa de reparto. Esto explica claramente por qué las placas funcionan perfectamente dentro de este tipo de tableros a pesar de su comportamiento frágil frente a este esfuerzo dominado por los criterios de prefisuración.

7) Dado el buen comportamiento a cortante por el efecto favorable del pretensado y contribución de la zona no fisurada junto al apoyo (mecanismo de cortante puro y criterio de tracción principal en el alma, 44.2.3.2.1.1 EHE), la continuación del trabajo se concentra en el estudio del comportamiento a rasante entre hormigones bajo la acción conjunta de la retracción, fluencia y cargas exteriores. En una primera etapa se está poniendo a punto un modelo de cálculo y está prevista una segunda etapa experimental concentrada en este fenómeno.

\section{REFERENCIAS}

(1) Pich-Aguilera, F., Batlle, T., Casaldáliga, P. (2008). La arquitectura residencial como una realidad industrial. Tres ejemplos. Informes de la Construcción, 6o(512): 47-60, doi:http://dx.doi.org/10.3989/ic.08.040.

(2) Salas, J. (2008). De los sistemas de prefabricación cerrada a la industrialización sutil de la edificación: algunas claves del cambio tecnológico. Informes de la Construcción, 6o(512): 19-34, doi: http://dx.doi.org/10.3989/ic.07.001.

(3) Manterola, J. (2006). Puentes. Apuntes para su diseño, cálculo y construcción. Madrid: Colección Escuelas.

(4) Ministerio de Fomento. (2008). Instrucción de Hormigón Estructural EHE-o8.

(5) Lundgren, K., Broo, H., Engström, B. (2004). Analyses of hollow core floors subjected to shear and torsion. Structural Concrete, 5(4): 161-172, doi: http://dx.doi.org/10.1680/stco.2004.5.4.161.

(6) López, A. (1990). Colección de tableros de puentes. Almería: Prefabricados J. Cirera Arcos S.A.

(7) CEN. (2005). EN1168, CEN/Tc229, European Standard EN 1168:2005 Precast concrete products - hollow core slabs. Comité Européen de Normalisation.

(8) CHALMERS - Civil and Environmental Engineering. (2004). Shear and torsion interaction of hollow core slabs. HOLCOTORS, Technical Report 3, Rev.1.

(9) Elliot, K.S., Davies, G., Bensalem, K. (1993). Precast Floor Slabs Diaphragms Without Structural Screeds. En Concrete 200 - Economic and Durable Construction Through Excellence (pp. 617-632).

(10) Ministerio de Fomento. (1998). IAP Instrucción sobre las acciones a considerar en el proyecto de puentes de carretera.

(11) Hernández, E., Gil, L. (2007). Hormigón Armado y Pretensado. Granada: Grupo de Investigación TEP-19o Ingeniería e Infraestructuras.

(12) Pajari, M. (2004). Pure Torsion Test on Single Slabs Units. Technical Research Centre of Finland. VTT Research notes 2273. 